\title{
Standard versUs peRForated (SURF) peripheral intravenous catheter trial: a pilot randomised controlled trial protocol
}

\author{
Nicole C Gavin, ${ }^{1,2,5}$ Nicole Marsh, ${ }^{1,2,5}$ Louise Marquart, ${ }^{3}$ Catherine O'Brien, ${ }^{2}$ Makrina Totsika, ${ }^{4}$ and Samantha Keogh ${ }^{* 1,2,5}$ \\ 'School of Nursing and Centre for Healthcare Transformation, Queensland University of Technology, QLD, Australia \\ ${ }^{2}$ Nursing and Midwifery Research Centre; Cancer Care Services; and Department of Medical Imaging; Royal Brisbane and Women's Hospital, \\ QLD, Australia \\ ${ }^{3}$ QIMR Berghofer Medical Research Institute QLD, Australia. \\ ${ }^{4}$ School of Biomedical Sciences and Centre for Immunology and Infection Control, Queensland University of Technology, QLD, Australia \\ ${ }^{5}$ Alliance for Vascular Access Teaching and Research (AVATAR), Menzies Health Institute Queensland, Griffith University, QLD, Australia
}

${ }^{*}$ Corresponding author: Professor Samantha Keogh, QUT School of Nursing, Victoria Park Road, Kelvin Grove, Brisbane, QLD 4059, Australia Email: sjkinoz@bigpond.net.au

Keywords contrast, CT scan, extravasation, peripheral intravenous catheter, randomised controlled trial, cancer

For referencing Gavin NC et al. Standard versUs peRForated (SURF) peripheral intravenous catheter trial: a pilot randomised controlled trial protocol. Vascular Access 2020; 6(2):9-14.

Dol https://doi.org/10.33235/va.6.2.9-14

\begin{abstract}
Introduction Contrast-enhanced computed tomography (CECT) scanning requires a large bore peripheral intravenous catheter (PIVC) to support the high-flow injection of contrast needed to generate a quality image. Unfortunately, not all patients' veins will tolerate high-flow injection and this can subsequently lead to complications, procedural delays, use of alternate intravascular access, and potential compromise of scan image. This study will evaluate the effectiveness of perforated catheters compared to standard catheters in reducing PIVC complications and failure during high-flow injection for CECT scans.
\end{abstract}

Methods and analysis This single-centre, parallel-group, pilot randomised controlled trial (RCT) will be undertaken to test the feasibility of conducting a larger trial comparing a PIVC with a novel perforated design to a standard (non-perforated) PIVC. The study will be conducted at the Royal Brisbane and Women's Hospital, Australia, and the recruitment target is 60 (30 per group). There are two primary outcomes of interest to test: 1) Feasibility of an adequately powered RCT with pre-established criteria for eligibility, recruitment, protocol adherence and retention; and 2) All cause PIVC failure rate. Secondary outcomes to be collected include first insertion success, subtypes of PIVC failure, PIVC dwell time, contrast delivery, and CECT image quality. Adverse and serious adverse events (i.e., extravasation, allergic reaction to the contrast) also will be monitored. Feasibility outcomes will be reported descriptively and assessed against pre-determined acceptability criteria. Statistical comparison methods will be tested. Regression analysis will assess the effect of patient and treatment differences.

Ethics and trial commencement This pilot trial has ethical approval from Queensland Health (HREC/2019/QRBW/55616) and Queensland University of Technology (QUTHREC/1900001090).

Trial registration ACTRN12619001780145 


\section{Strengths and limitations of the study}

- This is the first randomised controlled trial to explore the role of different PIVC designs on patient and CECT scan outcomes during CECT.

- Results of the pilot study will determine feasibility of study protocol for a larger trial.

- This pilot trial sample size is not large enough to detect the effectiveness of a perforated PIVC compared to a standard non-perforated PIVC on patient, device and scan outcomes.

\section{Introduction and background}

Peripheral intravenous catheters (PIVCs) are the most commonly used invasive medical device, with over 2 billion purchased each year globally and 25 million a year in Australia. ${ }^{1-3}$ Patients diagnosed with cancer are high end users of PIVCs and other vascular access devices, to receive both anti-cancer and adjunct treatments, using approximately 12.5 million PIVCs annually in Australia. ${ }^{4,5}$ Observational studies of vascular access devices in cancer care found that over $29 \%$ of patients required a PIVC and, of these, up to 50\% experienced complications (e.g., occlusion, infiltration and local infection) and failure..$^{5,6}$

Intravenous (IV) cytotoxic chemotherapy, rapid injection of radiopaque contrast, in addition to other drugs and infusates, are frequently required to be delivered via peripheral vascular access in cancer care services. These substances are known to be highly irritant to veins and frequently cause localised inflammation and sclerosis, which results in the loss of accessible veins. ${ }^{5.7}$ Consequently, difficult venous access during later stages of treatment and readmission are experienced by a significant proportion of patients. ${ }^{6.8}$ In some cases, central venous access - e.g., peripherally inserted central catheters (PICCs) or totally implanted vascular access devices (Portacaths) - may be established; however, this comes with increased cost and risks (i.e., infection). ${ }^{7}$ A number of strategies are employed to minimise IV complications such as heat compress, dilution and reduced rate of delivery. However, many of these lack an evidence base, are applied inconsistently, and unacceptable complication and failure rates persist. ${ }^{9}$ Vessel preservation and reduction of infection is a high priority in this vulnerable patient population.

Commercially, there is a plethora of differently designed PIVCS available to clinicians. However, other than the PIVCs' polymeric formulation, the invasive segment of the catheter has not changed in the last few decades. Traditional PIVCs are designed with a single end-hole at the distal tip of the catheter in which medications and IV fluids are administered. A relatively new innovative catheter design has recently become available that offers additional micro perforations on the side of the catheter (Nexiva ${ }^{\mathrm{TM}}$ Diffusics $^{\mathrm{TM}}$, BD Medical, Franklin Lakes, USA). It was specifically developed to facilitate high pressure contrast injection within the medical imaging setting. Research using computational models suggests that peripheral catheters with both distal and lateral holes are subject to lower shear forces, reduced wall shear stress, and lower pressure drop across the catheter, thus achieving more uniform radial velocities and flow distribution to each of the holes. ${ }^{10-12}$ A direct comparison of PIVC 18-gauge end-hole and side-hole catheters in a phantom model, observed with Schlieren imaging (of density variation), confirmed a reduction in velocity of contrast material exiting the tip and shear wall stress. ${ }^{13}$

Research on the effectiveness of the invasive component of the catheter design is limited to single, industry funded studies at present. ${ }^{13-15}$ The impact of the altered distribution of infusate due to catheter tip perforations on internal shear wall stress is theoretical and unexplored with this catheter design. Phlebitis, or more appropriately thrombophlebitis, itself is a poorly defined condition and the pathophysiology unclear. ${ }^{16}$ Rheology (flow) based investigations would be illuminating, but indicators of phlebitis (e.g., pain, redness, palpable cord) need to be observed among the clinical outcomes collected. Similarly, the potential impact of a perforated catheter tip on infection risk - local or bloodstream - is unknown. Theoretically, the perforations may have some relevance to biofilm formation (due to slight increase in surface area), but again this is unknown and untested. Microbiological testing in vitro and explanted also would be useful and add to the body of knowledge in this area. The noninvasive component of this innovative catheter, consisting of an integrated extension set with dual port access, has already demonstrated a significant reduction in phlebitis, improved dwell times, and a 20\% relative risk reduction in catheter related infection. ${ }^{17}$ This may be due to minimised handling and the closed system design. Rigorous, independent, large, multi-site trials testing the effectiveness of different catheter designs in broader and vulnerable populations is urgently required to inform policy makers and clinicians.

\section{Methods}

\section{Study design}

A single-centre, parallel-group, pilot randomised controlled trial (RCT) will be undertaken to test the feasibility of conducting a superiority RCT comparing a PIVC with a novel perforated design to a standard (non-perforated) PIVC. The trial has been designed and will be conducted in accordance with the Consolidated Standards of Reporting Trials (CONSORT) statement, ${ }_{1}^{18}$ and has been prospectively registered with the Australian New Zealand Clinical Trials Registry (ACTRN12619001780145).

\section{Ethics}

This study has ethical approval from Queensland Health (HREC/2019/QRBW/55616) and Queensland University of Technology (QUTHREC/1900001090). Written informed consent will be obtained from all study participants prior to randomisation. Data will be stored securely in a passwordprotected database and paper copies in a locked filing cabinet, 
as per the Australian National Health and Medical Research Council (NHMRC) guidelines. ${ }^{19}$

\section{Study aims, objectives and hypothesis}

The main aim and objective of this study is to test the feasibility of an RCT evaluating the impact of a novel perforated PIVC design compared to a standard (non-perforated) PIVC in patients undergoing cancer treatment and requiring high-flow IV administration of contrast. As a feasibility study, it is not powered for hypothesis testing, but the overarching clinical hypothesis is, The use of a perforated PIVC for injection of high-flow contrast will reduce the incidence of PIVC failure compared with the use of a non-perforated PIVC.

\section{Participants and setting}

A single-centre pilot RCT will be undertaken within Cancer Care Services and the Department of Medical Imaging at the Royal Brisbane and Women's Hospital (RBWH). Sixty patients (30 per treatment group) requiring injection of contrast through a PIVC to facilitate a computed tomography (CT) scan for diagnosis or staging of malignant oncology or haematology conditions will be enrolled.

Patients are eligible for inclusion if $\geq 18$ years of age and require a PIVC for injection of contrast as part of a cancer care diagnosis, prognosis, and/or treatment (outpatient or inpatient). They will be excluded if: the PIVC is inserted under emergency conditions; laboratory confirmed BSI (within previous 48 hours) exists; the patient is non-English speaking background (NESB) without an interpreter; the patient is receiving end-of-life care; there is a cognitive barrier to consent; the patient was previously enrolled in the study; or there is a known difficult IV access/ultrasoundguided placement regularly required.

\section{Sample size and statistical power}

All eligible patients will be screened consecutively. Sixty patients (30 per treatment group) will be recruited for the trial. The sample size is based on acquisition of feasibility outcomes rather than clinical outcomes. Thirty participants per treatment group is adequate to identify feasibility of study procedures and provide estimates of treatment effect for future investigations..$^{20,21}$

\section{Interventions}

Patients in the standard care group will receive an Insyte ${ }^{\mathrm{TM}}$ Autogard ВC $^{\text {тм }}$ (BD Medical) single end-hole (non-perforated) PIVC, with gauge as per the inserter's preference $(20 \mathrm{G} \times 30 \mathrm{~mm}$ or $22 \mathrm{G} \times 25 \mathrm{~mm})$. Participants allocated to the intervention group will have (as per clinician preference and assessment) BD Nexiva ${ }^{\mathrm{TM}}$ Diffusics $^{\mathrm{TM}}$ (BD Medical) end- and side-holes (with perforations) catheter inserted and used for infusion of contrast (20G X $31.75 \mathrm{~mm}$ or $22 \mathrm{G} \times 25.4 \mathrm{~mm})$.

\section{PIVC management}

Other than the study intervention (type of PIVC inserted), all other aspects of PIVC management will be as per local clinical guidelines (Royal Brisbane and Women's Hospital, 000259: Peripheral Intravenous Cannulation and Infusion Management - Adult and Paediatrics). Hand hygiene is required prior to and throughout the insertion procedure. Skin will be prepped and decontaminated using 2\% chlorhexidine gluconate with a $70 \%$ isopropyl alcohol swab (SoluPrep ${ }^{\text {TM }}$ Antiseptic Wipes, 3M, St Paul, MN, USA). The antiseptic must be allowed to dry prior to inserting the PIVC. Palpation of the insertion site should not be performed after the application of antiseptic, unless aseptic technique is maintained. If the health professional needs to re-establish the identification of the vein, the site should be re-prepped with the antiseptic solution and allowed to thoroughly dry. It is more efficient to assess the patient's veins at the outset, determine the degree of difficulty of insertion, and then risk assess to ascertain if it may be more effective to wear sterile gloves to enable palpating of the cleansed area, thereby maintaining aseptic nontouch technique (ANTT ${ }^{\oplus}$ ). Site selection will be determined by inserter, following assessment of the patient's vessel health and in consultation with radiographer/radiologist about procedure and infusion protocol.

Study and control PIVCs will be inserted by a trained research nurse (ReN) who is an existing skilled or competent PIVC inserter. Pre-trial, they will have training and simulated practice inserting the study PIVC until the ReN feels confident that their skills match their competence for the control PIVC insertion. Daily checks by ReN for protocol fidelity will be conducted if the study participants are in-patients.

A non-bordered, sterile, transparent, semi-permeable, self-adhesive IV dressing (Tegaderm, 3M St Paul, MN, USA) will be placed over the insertion site (as per manufacturer recommendations). The PIVC will be removed when prescribed therapy is complete or at 72 hours, in line with study site policy.

\section{Study outcomes}

In addition to patient and device characteristics, the following will be collected:

\section{Primary outcomes}

1. Study feasibility:22 The feasibility outcome will be determined based on the following criteria:

i. Eligibility: over $90 \%$ of screened patients meeting all inclusion and no exclusion criteria;

ii. Recruitment: over $90 \%$ of eligible patients providing informed consent (or not opting out);

iii. Retention: fewer than $5 \%$ of recruited patients lost to follow-up or withdrawing consent;

iv. Protocol fidelity: over $90 \%$ of randomised patients receiving their allocated intervention;

v. Missing data: less than $5 \%$ of primary endpoint data unable to be collected by study staff; and 
vi. Patient and staff acceptability with the study intervention and control: $70 \%$ of patients/parents and staff scoring $\geq 7$ on a 0-10 point rating scale at study completion.

2. PIVC failure: The PIVC failure outcome is a dichotomous variable (Yes/No), based on unplanned PIVC removal before the completion of prescribed therapy as a composite measure of the following complications at removal. This includes: occlusion (PIVC would not infuse, or leakage occurred around site when fluid injected); infiltration/extravasation (leaking of fluid into surrounding tissues); dislodgement (complete); phlebitis (defined by presence of one or more of the following symptoms: pain, redness, swelling and a palpable cord); and infection (local, primary or laboratory-confirmed bloodstream infection [BSI]). ${ }^{23} \mathrm{~A}$ composite measure of PIVC failure increases precision and efficiency and was chosen since PIVC failure is the outcome of importance to patients, with reduced patency taking various pathways to the same endpoint. ${ }^{24}$ This value is used to inform the sample size calculation for the subsequent larger study.

\section{Secondary outcomes}

Secondary outcomes are subtypes of PIVC failure (occlusion, infiltration/extravasation, dislodgement, phlebitis and infection) and PIVC dwell time (defined as time from insertion to removal). Additionally, first-time insertion success (Yes/No) and insertion pain (Numeric Rating Scale: $0=$ no pain to $10=$ extreme pain). For quality assurance, reporting of CECT image quality will be monitored (through region of interest (ROI) assessment). Adverse and serious adverse events (i.e., extravasation, allergic reaction to the contrast, primary or laboratory confirmed BSI within 48 hours, unplanned admission to the ward or intensive care unit, or death) will be monitored using hospital records and reported to the Human Research Ethics Committee at the study site.

\section{Microbiological testing and endpoints}

To further inform the infection outcomes, a sub-study evaluating microbial colonisation and biofilm formation will be conducted..$^{25}$ Microorganisms will be isolated and identified according to standard hospital protocol. Tip cultures will be considered colonised if growth is $\geq 15$ colonising forming units (CFU). Blood cultures (if ordered by clinicians) from a peripheral vein will be cultured by Microbiology Pathology Queensland - Central Lab to provide differential time to positivity BSI diagnosis, and the pathology results downloaded. The microbial biofilms on a purposive random sample (10-20\%) of PIVCs will be evaluated using next generation sequencing (MiSeq Illumina). Briefly, microbial DNA will be extracted and amplified using barcoded primers targeting hypervariable regions (V5-V7) of the 165 rRNA gene and then sequenced unidirectionally using the Illumina MiSeq System. The sequences will be analysed using QIIME and $R$ software. The results will add value and assist interpretation of the impact of different catheter design on the PIVC colonisation and/or BSI.

\section{Screening, recruitment and consent}

Department of Medical Imaging lists will be screened daily (Monday-Friday) by the ReN to assess for eligible patients. After consultation with clinicians, eligible patients will be approached. Study procedures will be explained to the participants, a written information sheet will be provided, and the ReN will answer any questions the patient has about their involvement in the study. Eligible and consenting patients will be enrolled in the study.

\section{Randomisation and allocation concealment}

Eligible and consenting participants will be randomised to treatment allocation by the ReN using a secure web-based central randomisation service. The allocation sequence will be computer-generated (Griffith University Trial Randomisation Service) in a ratio of 1:1, using a randomly variable permuted block size $(2,4$ and 6$)$ to avoid allocation prediction. The intervention is not amenable to blinding of patients, clinical staff nor ReNs. However, radiologists assessing image quality, physicians assessing infection, microbiologists assessing colonisation, and the data analyst will be blinded to group allocation.

Daily screening reports will be stored and de-identified records entered into an electronic Excel spreadsheet. Screening reports provide the total number of patients screened, number of patients excluded with reasons, and the number of patients included, randomised, and lost to follow-up.

\section{Data collection}

Data will be collected by ReNs on a study specific electronic case report form (CRF) in REDCap (Research Electronic Data CAPture, Vanderbilt). ${ }^{26,27}$ All clinical study data as detailed in outcomes section and PIVC failure will be collected and managed using REDCap electronic data tools hosted at Queensland University of Technology (QUT). REDCap is a secure, web-based application designed to support rapid data capture providing an intuitive interface for validated data entry, audit trails for tracking data manipulation and export procedures, and automated export procedures for seamless data downloads to common statistical packages. The primary investigator will liaise with the ReN weekly to undertake quality checks for allocation integrity and data verification. Upon trial completion, only the chief/principal investigators and the ReN will have access to the de-identified data (once exported).

At enrolment, the ReN will collect data on patient demographics plus clinical, device and procedural factors. At device removal, the ReN will record the reason for device removal, site condition and outcome of procedure. The patient's condition and that of their PIVC insertion site will be checked with a routine followup telephone call at 48 hours post-removal if discharged or outpatient. Patients who remain in hospitals will have a site inspection performed by the ReN. 


\section{Statistical methods}

Feasibility outcomes will be reported descriptively. All randomised patients will be analysed on an intention-to-treat basis. Patients will have one PIVC entered in the study so that the unit of analysis (the patient) is independent. For this pilot trial, we will test the feasibility of the statistical analysis that will be used in the definitive trial. Prior to analysis, data will be cleaned and checked. All attempts will be made to collect the primary endpoint PIVC failure (Yes/No). Data will be exported to Stata 16 (College Station, TX: StataCorp LLC). Comparability of groups at baseline will be assessed using clinically relevant indicators and compared statistically using chi-squared tests to compare differences in categorical variables and independent samples t-tests to compare differences in continuous variables. Frequency and incidence rate ratio (with 95\% confidence intervals) of device failure will summarise the impact of the intervention. Secondary endpoints will be compared between groups for clinically significant differences, with the impact of the intervention on dwell times assessed using hazard ratio (with 95\% confidence intervals) estimated from a Cox proportional hazards model and the impact of the intervention on categorical variables assessed by frequency and incidence rate ratios (with 95\% confidence intervals). $p$-values of $<0.05$ will be considered statistically significant.

\section{Validity and reliability}

Strategies employed to maintain internal validity for this trial include the following: web-based randomisation and allocation concealment until randomisation; blinding of the study statistician, microbiologist, radiologist and infectious diseases physician; and all patients randomised will be accounted for by using an intention-to-treat approach. External validity for this study will be improved by clearly defining the characteristics of the target population and inclusion and exclusion criteria. Reliability will be assured by use of a single inserter and expert vascular access data collector, plus double entry and checking of $10 \%$ of data.

\section{Discussion}

This research has the potential to improve health service delivery and the patients' PIVC experience within medical imaging. This will be the first independent trial to evaluate novel PIVC design on patient and imaging outcomes. There is limited research investigating PIVC designs and safety generally, inclusive of departments of medical imaging. Further, the impact of the PIVC plus infusion or injection of fluids on vessel endothelium and blood componentry is poorly understood. In addition to testing the impact of an innovative catheter design, this research will yield new knowledge to enhance our understanding of vascular access devices in modern healthcare. The perforated design of the catheter tip may be the key to reducing PIVC failure more broadly through its 'diffusive' action in vivo, minimising endothelial and platelet activation from repeated injection and infusion in everyday use. The merits of a comprehensive integrated, closed system and perforated design of the catheter may reduce the risk of infiltration/extravasation and infection. Knowledge of this could result in a paradigm shift in PIVC design, insertion, and use.

\section{Disclosures}

NM: Griffith University has received on her behalf investigatorinitiated research grants and unrestricted educational grants from Becton Dickinson and Cardinal Health, and a consultancy payment provided to Griffith University from Becton Dickinson (unrelated to the current project). SK's employer (QUT) has received monies on her behalf from $\mathrm{BD}$ Medical for educational consultancies, outside the submitted work. All other authors have nothing to declare.

\section{Funding}

This study is funded by the 2018/9 Metro North Hospital and Health Service Collaborative Research Grant ref: CRG191-2019.

\section{References}

1. Alexandrou E, Ray-Barruel G, Carr PJ, Frost SA, Inwood S, Higgins N, et al. Use of short peripheral intravenous catheters: characteristics, management, and outcomes worldwide. J Hosp Med 2018;13(5). doi:10.12788/jhm.3039.

2. Australian Institute of Health and Welfare. Australian hospital statistics 2017-2018; 2018. Available from: https://www.myhospitals.gov. au/hospital/310000011/princess-alexandra-hospital/emergencydepartment

3. Tuffaha H, Marsh N, Byrnes J, Gavin N, Webster J, Cooke M, et al. Cost of vascular access devices in public hospitals in Queensland. Aust Health Rev 2018 Sept 4. doi:10.1071/AH18102.

4. Cancer Australia. Cancer Australia statistics; 2018. Available from: https:// canceraustralia.gov.au/affected-cancer/what-cancer/cancer-australiastatistics.

5. Russell E, Chan RJ, Marsh N, New K. A point prevalence study of cancer nursing practices for managing intravascular devices in an Australian tertiary cancer center. Eur J Oncol Nurs 2014 Jun;18(3):231235. doi:10.1016/j.ejon.2013.11.010.

6. Larsen EN, Marsh N, O'Brien C, Monteagle E, Friese C, Rickard C. Inherent and modifiable risk factors for peripheral venous catheter failure during cancer treatment: a prospective cohort study. Support Care Cancer 2020 Jul 24. doi:10.1007/s00520-020-05643-2.

7. Gallieni M, Pittiruti M, Biffi R. Vascular access in oncology patients. CA Cancer J Clin 2008 Oct; 58(6):323-346. doi:10.3322/CA.2008.0015.

8. LeVasseur N, Stober C, Ibrahim M, Gertier S, Hilton J, Robinson A, et al. Perceptions of vascular access for intravenous systemic therapy and risk factors for lymphedema in early-stage breast cancer-a patient survey. Curr Oncol 2018 Aug; 25(4):e305-e310. doi:10.3747/co.25.3911.

9. Ding S, Meystre NR, Campeanu C, Gullo G. Contrast media extravasations in patients undergoing computerized tomography scanning: a systematic review and meta-analysis of risk factors and interventions. JBI Database System Rev Implement Rep 2018 Jan;16(1):87-116. doi:10.11124/JBISRIR-2017-003348.

10. Daniel TB, Akins EW, Hawkins IF. A solution to the problem of high-flow jets from miniature angiographic catheters. AJR Am J Roentgenol 1990 May;154(5):1091-1095. doi:10.2214/ajr.154.5.2108548. 
11. Grigioni M, Daniele C, Morbiducci U, et al. Computational model of the fluid dynamics of a cannula inserted in a vessel: incidence of the presence of side holes in blood flow. J Biomech 2002 Dec;35(12):15991612.

12. Park JY, Park CY, Min BG. A numerical study on the effect of side hole number and arrangement in venous cannulae. J Biomech 2007;40(5):1153-1157. doi:10.1016/j.jbiomech.2006.04.005.

13. Weber PW, Coursey CA, Howle LE, Nelson RC, Nichols EB, Schindera ST. Modifying peripheral IV catheters with side holes and side slits results in favorable changes in fluid dynamic properties during the injection of iodinated contrast material. AJR Am J Roentgenol 2009 Oct;193(4):970977. doi:10.2214/AJR.09.2521.

14. Johnson PT, Christensen GM, Fishman EK. I.V. contrast administration with dual source 128-MDCT: a randomized controlled study comparing 18-gauge nonfenestrated and 20-gauge fenestrated catheters for catheter placement success, infusion rate, image quality, and complications. AJR Am J Roentgenol 2014 Jun;202(6):1166-1170. doi:10.2214/AJR.13.11730.

15. Hasapes JP. Effect of three side hole infusion catheter on CT image quality compared to a standard infusion catheter. ClincalTrials.gov, 2016.

16. Ray-Barruel G, Polit DF, Murfield JE, Rickard CM. Infusion phlebitis assessment measures: a systematic review. J Eval Clin Pract 2014 Apr;20(2):191-202. doi:10.1111/jep.12107.

17. Gonzalez Lopez JL, Arribi Vilela A, Fernandez del Palacio E, Olivares Corrai J, Benedictio Marti C,Herrera Portal P. Indwell times, complications and costs of open vs closed safety peripheral intravenous catheters: a randomized study. J Hosp Infect 2014 Feb;86(2):117-126. doi:10.1016/j. jhin.2013.10.008.

18. Eldridge SM, Chan CL, Campbell MJ, Bond CM, Hopewell S, Thabane $L$, et al. CONSORT 2010 statement: extension to randomised pilot and feasibility trials. BMJ 2016;355:i5239. doi:10.1136/bmj.i5239.

19. NHMRC. National statement on ethical conduct in human research. In: NHMRC (ed.). Canberra: Commonwealth of Australia; 2018.

20. Hertzog MA. Considerations in determining sample size for pilot studies. Res Nurs Health 2008 Apr;31(2):180-191. doi:10.1002/nur.20247.

21. Whitehead $\mathrm{AL}$, Julious $\mathrm{SA}$, Cooper $\mathrm{CL}$, Campbell MJ. Estimating the sample size for a pilot randomised trial to minimise the overall trial sample size for the external pilot and main trial for a continuous outcome variable. Stat Methods Med Res 2016 Jun;25(3):1057-1073. doi:10.1177/0962280215588241

22. Thabane L, Ma J, Chu R, Cheng J, Ismaila A, Rios LP, et al. A tutorial on pilot studies: the what, why and how. BMC Med Res Methodol 2010 Jan;10(1):1. doi:10.1186/1471-2288-10-1.

23. CDC. National Safety Network (NHSN) patient safety component manual. Atlanta, USA: Department of Health and Human Services; 2018.

24. Freemantle N, Calvert M, Wood J, Eastaugh J, Griffin C. Composite outcomes in randomized trials: greater precision but with greater uncertainty? JAMA 2003 May;289(19):2554-2559. doi:10.1001/ jama.289.19.2554

25. Maki DG, Weise CE, Sarafin HW. A semiquantitative culture method for identifying intravenous-catheter-related infection. N Engl J Med 1977 Jun 9;296(23):1305-1309. doi:10.1056/NEJM197706092962301.

26. Harris PA, Taylor R, Minor BL, Elliott V, Fernandez M, O'Neal L, et al. The REDCap consortium: building an international community of software platform partners. J Biomed Inform 2019 Jul;95:103208. doi:10.1016/j. jbi.2019.103208.

27. Harris PA, Taylor R, Thielke R, Payne J, Gonzalez. N, Conde J. G. Research electronic data capture (REDCap): a metadata-driven methodology and workflow process for providing translational research informatics support. J Biomed Inform 2009 Apr;42(2):377-381. doi:10.1016/j. jbi.2008.08.010. 